\title{
Isolated Proximal Leg Paresis due to a Small Cortical Infarction
}

\author{
Kazuyuki Noda ${ }^{1}$, Momo Tani ${ }^{2}$, Jiro Fukae ${ }^{2}$, Kenji Fujishima ${ }^{1}$, \\ Nobutaka Hattori ${ }^{2}$ and Yasuyuki Okuma ${ }^{1}$
}

\begin{abstract}
We describe two patients with small cortical infarcts, who presented with isolated proximal weakness in one of their legs. These lesions were located in the contralateral precentral gyrus, more medial than the precentral knob, but more lateral than the topmost part of the motor cortex. These clinical findings are consistent with the physiological findings of Penfield and Boldrey, and those of recent activation studies by functional MRI. It is clinically important to pay close attention to the contralateral top of the motor cortex when examining pure motor monoparesis of a proximal part of a lower extremity.
\end{abstract}

Key words: leg paresis, cortical infarction, primary motor cortex, MRI

(Inter Med 49: 1633-1636, 2010)

(DOI: 10.2169/internalmedicine.49.3533)

\section{Introduction}

Recent advances in functional imaging have led to a renewed interest in the cortical mapping of various body parts. To accurately identify the anatomical localization and elucidate the function of the primary motor cortex (M1), it is important to identify lesions and examine the signs and symptoms of patients with cortical infarction presenting with pure motor monoparesis (PMM). However, PMM limited to one lower extremity caused by a cortical infarction is rare $(1,2)$. In a large series of 1,575 patients with an acute stroke, 63 patients $(4 \%)$ were found to have a leg-predominant weakness, and only two of whom showed PMM in the proximal part of a leg (3). In addition, two separate case reports showed PMM in the distal part of a leg $(4,5)$. Here, we describe two patients who presented with proximal weakness in a lower extremity caused by a small cortical infarction.

\section{Case Report}

\section{Case 1}

An 84-year-old right-handed man with a history of hyper- tension was admitted because of sudden-onset weakness of his right leg making walking difficult. The patient did not complain of lower back pain. He did not notice weakness on his face or of his right upper extremity. On admission, his temperature was $36.4^{\circ} \mathrm{C}$ and his pulse was 72 beats per minute. His blood pressure was $156 / 72 \mathrm{mmHg}$. General physical examination showed normal findings. On neurologic examination, he was alert and oriented. There was no aphasia, agnosia, or apraxia. His cranial nerves were all intact. Motor examination revealed mild weakness (Medical Research Council [MRC] grade, 5-/5) of the right iliopsoas and quadriceps femoris. The knee flexors, the distal part of the right leg, and the right shoulder next to thigh motions in the motor homunculus, showed normal strength. The rest of the motor examination findings were normal. There was no muscular atrophy in both proximal and distal muscles bilaterally. Deep tendon reflexes (DTRs) were within normal limits, and no pathological reflexes were observed. His coordination and sensory systems were normal. Hematological tests and urinalysis showed normal findings. The findings of the coagulation function tests were normal. Blood chemistry revealed mild elevation of serum creatinine $(1.4 \mathrm{mg} / \mathrm{dL})$, blood glucose $(112 \mathrm{mg} / \mathrm{dL})$, and triglyceride $(220 \mathrm{mg} / \mathrm{dL})$ levels. All other laboratory tests showed normal findings.

${ }^{1}$ Department of Neurology, Juntendo University Shizuoka Hospital, Izunokuni and ${ }^{2}$ Department of Neurology, Juntendo University School of Medicine, Tokyo

Received for publication February 14, 2010; Accepted for publication April 26, 2010

Correspondence to Dr. Kazuyuki Noda, k-noda@juntendo.ac.jp 

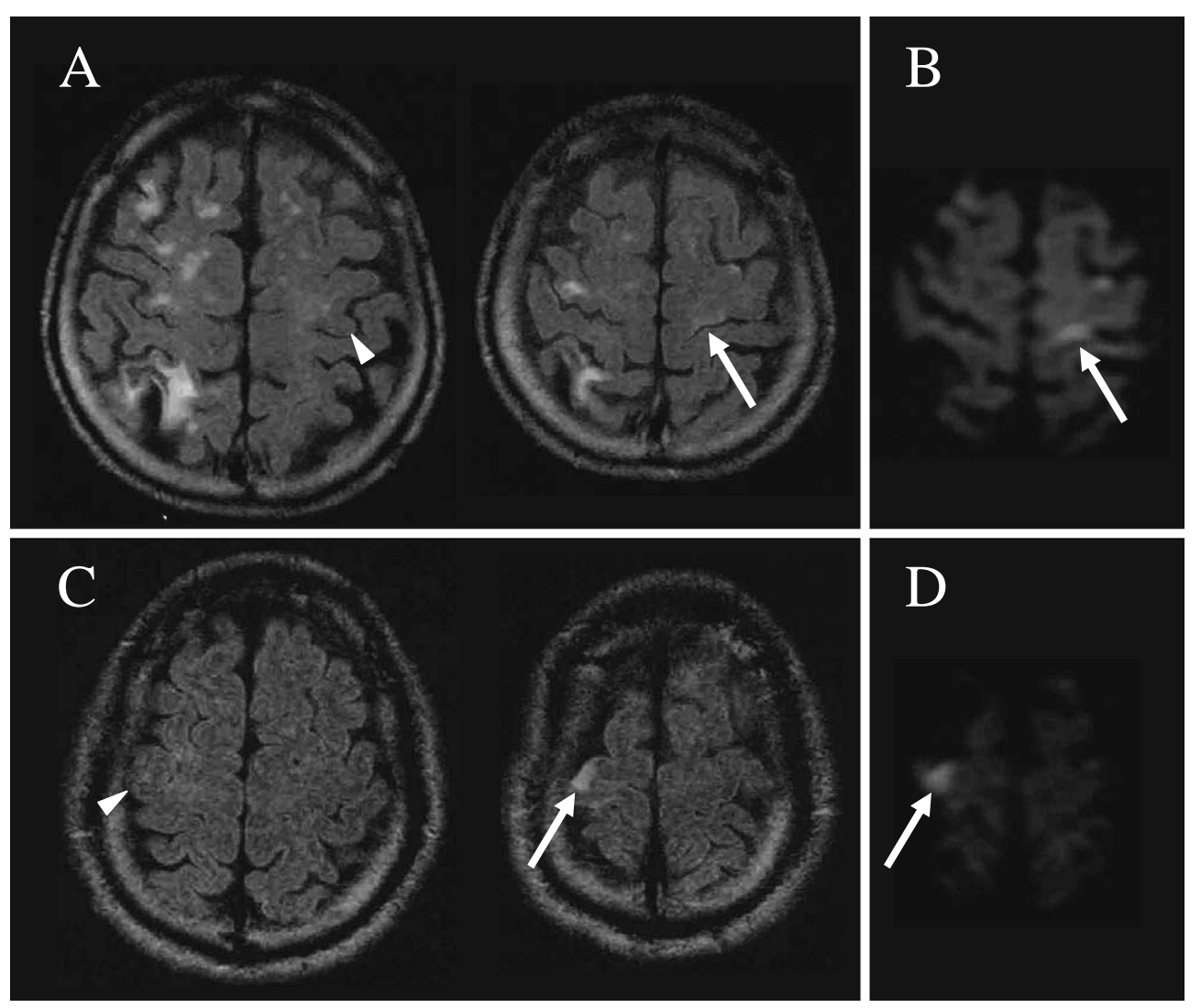

Figure 1. MRI images of brain of Case $1(A, B)$ and Case $2(C, D)$. Fluid-attenuated inversion recovery images $(A, C)$ and diffusion-weighted images $(B, D)$ show the motor cortical infarctions in the precentral gyrus (arrows). Arrowheads indicate the precentral knob presenting the motor hand area.

His glycated hemoglobin (HbA1c) level was 5.6\%. An electrocardiogram showed a sinus rhythm at a rate of 75 beats per minute without specific ST-segment and T-wave abnormalities. Plain radiographs of the cervical and lumbar spine showed no narrowing of the spinal canal or "vacuum phenomenon" at the intervertebral disc spaces. No displacement or malalignment of vertebral bodies was observed. Brain MRI with fluid-attenuated inversion recovery imaging (FLAIR), performed 4 hours after the onset, showed high signal-intensity areas in the right hemisphere and the medial part of the left precentral gyrus. Brain MRI with diffusionweighted imaging (DWI) showed a small high signalintensity area located in the medial part of the left precentral gyrus, which is indicative of infarction in the acute stage (Fig. 1A, 1B). There were no identifiable lesions in the corona radiata, internal capsule, cerebral peduncle, and pontine base. Apparent diffusion coefficient (ADC) maps were not examined. Cranial MR angiography (MRA) revealed atherosclerotic changes in both the internal carotid arteries. Given the presence of hypertension and the acute onset of leg weakness, he was diagnosed as having an atherothrombotic cerebral infarction with artery-to-artery embolism. Therefore, $180 \mathrm{mg} /$ day sodium ozagrel (antiplatelet agent) was started. After the initiation of the treatment, the symptom did not worsen. In addition, he was administered aspirin $(100 \mathrm{mg} /$ day) and ticlopidine (200 mg/day) orally to prevent further ischemic events. The patient was discharged from the hospital with mild improvement of his right leg disability on day 11.

\section{Case 2}

A 68-year-old right-handed man, who is a smoker with a history of hypertension developed acute proximal weakness in his left leg. The patient did not complain of lower back pain. He noticed heaviness of his left leg while walking and visited our hospital 3 days after the onset. His temperature was $35.3^{\circ} \mathrm{C}$ and his pulse was 67 beats per minute. His blood pressure was 126/86 mmHg. General physical examination revealed normal findings. On neurologic examination, he was alert and oriented. There was no aphasia, agnosia, or apraxia. His cranial nerves were all intact. Motor examination revealed mild weakness of the left leg (iliopsoas, 4/5, quadriceps femoris, 5-/5). The knee flexors, distal part of the left leg, and left shoulder showed normal strength. The rest of the motor examination findings were normal. There was no muscular atrophy in both proximal and distal muscles bilaterally. DTRs were normal, and no pathological reflexes were observed. His coordination and sensory systems were normal. Hematological tests and urinalysis showed normal findings. Blood chemistry revealed mild elevation of triglyceride level $(212 \mathrm{mg} / \mathrm{dL})$. All other laboratory tests showed normal findings. His HbAlc level was $4.8 \%$. An 
electrocardiogram showed a sinus rhythm at a rate of 63 beats per minute without specific ST-segment and T-wave abnormalities. Plain radiographs of the cervical and lumbar spine showed no narrowing of the spinal canal or "vacuum phenomenon" at the intervertebral disc spaces. No displacement or malalignment of vertebral bodies was observed. MRI with FLAIR and DWI 3 days after the onset revealed a recent small cortical infarction located in the medial part of the right precentral gyrus (Fig. 1C, 1D). ADC maps were not examined. Cranial MRA revealed mild atherosclerotic changes of anterior cerebral arteries and middle cerebral arteries bilaterally. Given the presence of hypertension, smoking history, and the acute onset of leg weakness, he was diagnosed as having an atherothrombotic cerebral infarction with artery-to-artery embolism. An oral administration of aspirin (100 mg/day) was initiated, and the weakness of his left leg gradually improved within two weeks.

\section{Discussion}

Clinical and neuroradiologic characteristics of these two patients were essentially the same. The common manifestation in both patients was PMM in the proximal part of the lower extremities. Isolated proximal leg paresis can be caused by a lesion in M1, the posterior limb of the internal capsule (PLIC), spinal cord or the peripheral nerves. Our patients showed no upper or lower motor neuron signs to help localization. There was no sensory loss indicating radiculopathy or spinal cord involvement. Because no abnormalities were found in their lumbar spines, radiculopathy seems to be less likely, although electromyography was not performed. Their brain MRI with DWI indicated a new infarction involving the topographically corresponding M1, although ADC maps to exclude false-positive results caused by the $\mathrm{T} 2$ shine-through effect were not available. Because the right-side hyperintense lesions on FLAIR of case 1 did not show hyperintensity on DWI, a high signal-intensity area on DWI located in the left M1 indicated a new lesion. Recently, Hiraga et al reported that PMM of the leg can be caused by an infarction of PLIC or corona radiata (6). However, MRI with DWI of the present patients revealed no lesions in PLIC and corona radiata. Therefore, small discrete cortical infarctions involving the top of M1 were most likely responsible for the motor disturbances in our patients. Regarding the pathogenesis of stroke in our patients, they showed atherosclerotic changes in internal carotid arteries and their branches bilaterally, but they did not show atrial fibrillation, one of the most prevalent causes of cardiogenic cerebral embolism (7). Therefore, considering the findings of cranial MRA and the mode of onset, our patients were considered to have artery-to-artery embolism as the underlying pathogenic mechanism.

In our patients, the degree of motor impairment was mild. This finding provides us a further possibility. Hemiparesis caused by upper motor lesions is more detrimental to the distal rather than the proximal body musculature (8). There is evidence that motor neurons innervating axial and proximal limb muscles receive both ipsilateral and contralateral descending inputs, whereas peripheral muscles are supplied by motor neurons, whose supraspinal inputs are mainly contralateral in monkeys $(9,10)$ and humans $(11)$. Therefore, it is conceivable that the symptom of our patients was mild because of these well-documented corticospinal connections. However, further studies of not only corticospinal projections, but also other factors such as the delimiting size of a particular brain lesion (12) and medical conditions, are required to determine the degree of motor weakness caused by cortical lesions.

These small infarctions were located in the contralateral precentral gyrus, more medially than the precentral knob, which is a reliable landmark of the motor hand area (13). The lesions were however located more laterally than the topmost part of the precentral gyrus, where Kohno et al (4) and Cattaneo et al (5) clearly detected a lesion in the case of isolated distal leg paresis. Using electrical stimulation of the brain surface during surgery, Penfield and Boldrey showed that the origin of the motor innervation of the proximal part of the leg is located between the areas of the distal part of the leg and arm (14). Our clinical findings confirm the results of Penfield and Boldrey's study. Recently, functional MRI studies to map the somatotopic organization of M1 have shown that the M1 subdivisions of the lower extremity medial to the hand are evident (15). Moreover, Kapreli et al found that contralateral M1 activations produced with the movements of the joints in the knee, ankle, and toes showed a somatotopic organization; the clearest shift in alignment was found in the lateral-to-medial direction, with the knee, ankle, and toe activations shifting consistently from lateral to medial (16). The data is consistent with the clinical findings in the present patients and also in the patients of Kohno et al (4) and Cattaneo et al (5).

In conclusion, PMM of a lower extremity caused by M1 lesions is rare. It is clinically important to pay close attention to the contralateral top of M1, when examining PMM of a lower extremity.

\section{References}

1. Melo TP, Bogousslavsky J, van Melle G, Regli F. Pure motor stroke: a reappraisal. Neurology 42: 789-795, 1992.

2. Paciaroni M, Caso V, Milia P, et al. Isolated monoparesis following stroke. J Neurol Neurosurg Psychiatry 76: 805-807, 2005.

3. Schneider R, Gautier JC. Leg weakness due to stroke. Site of le- sions, weakness patterns and causes. Brain 117: 347-354, 1994.

4. Kohno Y, Ohkoshi N, Shoji S. Pure motor monoparesis of a lower limb due to a small infarction in the contralateral motor cortex. Clin Imaging 23: 149-151, 1999.

5. Cattaneo L, Cucurachi L, Pavesi G. Isolated toe paralysis caused 
by a small cortical infarction. J Neurol Neurosurg Psychiatry 80: 1142,2009

6. Hiraga A, Uzawa A, Tanaka S, Ogawara K, Kamitsukasa I. Pure monoparesis of the leg due to cerebral infarctions: a diffusionweighted imaging study. J Clin Neurosci 16: 1414-1416, 2009.

7. Page RL. Newly diagnosed atrial fibrillation. N Engl J Med 351: 2408-2416, 2004

8. Adams RW, Gandevia SC, Skuse NF. The distribution of muscle weakness in upper motoneuron lesions affecting the lower limb. Brain 113: 1459-1476, 1990.

9. Lacroix S, Havton LA, McKay $\mathrm{H}$, et al. Bilateral corticospinal projections arise from each motor cortex in the macaque monkey: a quantitative study. J Comp Neurol 473: 147-161, 2004.

10. Kuypers HG. Some aspects of the organization of the output of the motor cortex. Ciba Found Symp 132: 63-82, 1987.

11. Blumenfeld $\mathrm{H}$. Corticospinal tract and other motor pathways. In: Neuroanatomy Through Clinical Cases. 1st ed. Sinauer Associates Inc., Sunderland, 2002: 213-261.
12. Chen CL, Tang FT, Chen HC, Chung CY, Wong MK. Brain lesion size and location: effects on motor recovery and functional outcome in stroke patients. Arch Phys Med Rehabil 81: 447-452, 2000 .

13. Yousry TA, Schmid UD, Alkadhi H, et al. Localization of the motor hand area to a knob on the precentral gyrus. A new landmark. Brain 120: 141-157, 1997.

14. Penfield W, Boldrey E. Somatotopic motor and sensory representation in the cerebral cortex of man as studied by electrical stimulation. Brain 60: 389-443, 1937.

15. Lotze M, Erb M, Flor H, Huelsmann E, Godde B, Grodd W. fMRI evaluation of somatotopic representation in human primary motor cortex. Neuroimage 11: 473-481, 2000.

16. Kapreli E, Athanasopoulos S, Papathanasiou M, et al. Lower limb sensorimotor network: issues of somatotopy and overlap. Cortex 43: 219-232, 2007.

(C) 2010 The Japanese Society of Internal Medicine http://www.naika.or.jp/imindex.html 